\title{
Developing a Systematic Search Strategy Related to People with Disability: A Test of the Utility of Proposed Disability Search Terms in a Search about Opioid Use
}

\author{
Michael Ioerger, Reed M. Flanders, Katherine D. Goss, Margaret A. Turk \\ SUNY Upstate Medical University
}

PREPRINT

OSF Preprint: July 6, 2018

\begin{abstract}
Author Note
Michael Ioerger, PhD, MPH, Department of Physical Medicine \& Rehabilitation, SUNY Upstate Medical University, Syracuse, NY 13244, USA; Reed M. Flanders, B.A., Department of Physical Medicine \& Rehabilitation, SUNY Upstate Medical University, Syracuse, NY 13244, USA; Katherine D. Goss, B.S., Department of Physical Medicine \& Rehabilitation, SUNY Upstate Medical University, Syracuse, NY 13244, USA; Margaret A. Turk, MD, Department of Physical Medicine \& Rehabilitation, SUNY Upstate Medical University, Syracuse, NY 13244, USA.

Correspondence concerning this article should be addressed to Michael Ioerger, 304 Jacobsen Hall, Department of Physical Medicine \& Rehabilitation, SUNY Upstate Medical University, Syracuse, NY 13210. Phone: 315-464-4782. Fax: 315-464-8674. E-mail:

ioergerm@upstate.edu.
\end{abstract}

\begin{abstract}
Background: The varied use of the term "disability" in the scientific literature makes it challenging to conduct systematic searches and reviews of health issues among people with disability. Given these challenges, utilizing general disability search terms, instead of condition-specific search terms, has been put forward as an efficient and effective way to ensure a broad capture of the literature related to disability when conducting a systematic search.

Objectives: This study evaluates the utility of general disability terms, as opposed to conditionspecific terms, in the context of systematically searching for sources related to disability, and in this case, opioid use.

Methods: Systematic searches were conducted utilizing three different databases (i.e., Medline, CINAHL, PsycINFO). An initial search of sources mentioning opioids and disability was conducted utilizing the general search terms recommended by Walsh et al. ${ }^{2}$ The results of this search were then compared to those of each of the 16 condition-specific searches. The proportion of unique sources from each condition-specific search that overlapped with the general search were assessed.

Results: There was very little overlap between the sources captured using condition-specific search terms and the sources captured utilizing the general search terms. The highest amount of overlap was for spinal muscular atrophy at $33.3 \%$, with the overall median proportion of overlap being $13.4 \%$ $($ mean $=15.7 \%$; SD $=11.7 \%)$.

Conclusions: With a systematic search for manuscripts about disability associated with opioid use as an example, condition-specific search terms capture a large proportion of sources not captured using general disability search terms. Disability researchers should be aware of pitfalls using general terminology and the importance of using disability-specific search terms, depending on the focus of the search. Implications for disability-focused systematic searches are discussed.

Keywords: Opioid, Disability, Literature Search
\end{abstract}




\section{Developing a Systematic Search Strategy Related to People with Disability: A Test of the Utility of Proposed Disability Search Terms in a Search about Opioid Use}

Systematic search and reviews have become the foundation for development of evidencebased practice and guideline development. In particular, evidence focused on the health issues of people with disability is scattered and more limited when compared to publications about health in the general public or for specific high prevalence conditions. Thus, systematic searches and reviews are of critical importance when trying to collect the extant knowledge about health and interventions related to people with disability. Opioid use in people with disability is one such health issue. The opioid crisis has garnered a lot of attention in the scientific literature because of its public health implications. However, there is seemingly little known about opioid use among people with intellectual and physical disability. In order to elucidate the current scientific understanding of this topic and identify knowledge gaps, a systematic search and review is needed. However, because of the various uses of the term "disability" in the scientific literature, identifying search terms for this kind of systematic search poses a substantial challenge. ${ }^{1}$

This study set out to evaluate the utility of using the disability search terms proposed by Walsh et al. ${ }^{2}$ to capture articles mentioning opioid and intellectual or physical disability as part of a systematic search. The results using these search terms were compared to the results of searches utilizing condition-specific search terms (e.g., spinal cord injury, cerebral palsy, autism) across three databases. The amount of overlap of unique sources between the recommended general search terms and each of the condition-specific search terms was evaluated.

\section{Method}

\section{Search Protocol}

A systematic search of three databases (Medline, CINAHL, PsycINFO) was conducted in 
June 2018. Initially, each database was searched by crossing the general disability search terms proposed by Walsh et al. ${ }^{2}$ with the opioid search terms (see Table 1). Then additional searches were conducted for all three of the databases crossing each of 16 condition-specific search terms (see Table 1) with the opioid search terms (see online Supplemental Materials - Appendix A for full search strategy details). There were no date restrictions, but a limiter was applied to restrict results to journal articles. This process yielded results from 51 individual searches. These results were then combined into a single file and coded to identify the searches and databases in which each unique source was found.

\section{Data Analysis}

Analyses focused on the proportion of unique sources found using the condition-specific search terms that were also found using the general search terms (across the three databases). The mean, standard deviation, and median were calculated.

\section{Results}

\section{Search Results}

A total of 9052 results were returned from 3 different databases (Medline $=4,664$, CINAHL $=1,947$, PsycINFO $=2,441$; see online Supplemental Materials - Appendix B for an analysis of database overlap) using our search protocol. From these results, there were 4623 unique sources. The difference between the number of total returned results and the total unique sources represents finding specific sources multiple times either with different search terms, different databases, or both. The total number of unique sources found for each specific search are provided in Table 2. These numbers represent the total number of unique articles that would have been found across the three databases only using that single search.

\section{Search Term Overlap}


The majority of the sources identified through the condition-specific searches were not captured using the general disability search terms proposed by Walsh et al. (see Figure 1). The highest proportions of overlap between the search utilizing the general disability terms and condition-specific searches were found for spinal muscular atrophy $(33.3 \%)$, concussions (30.0\%), and ADHD (28.3\%). The lowest proportions of overlap were found for spina bifida $(0.0 \%)$, muscular dystrophy $(0.0 \%)$, and ALS $(4.8 \%)$. The mean proportion of overlap was $15.7 \%(\mathrm{SD}=11.7 \%)$, with $13.4 \%$ representing the median.

\section{Discussion}

The varied use of the term "disability" in the scientific literature makes it challenging to conduct systematic searches related to health issues that impact people with disability. ${ }^{1}$ To address this issue, Walsh et al. ${ }^{2}$ has proposed a set of general search terms to capture sources in the scientific literature related to disability. This study found that these general disability terms captured only a small number of articles related to specific disability conditions in the context of identifying sources mentioning opioids. This suggests that systematic searches exploring health topics, such as opioid use, among people with disability would need to utilize condition-specific search terms in order to ensure a capture of the potentially relevant scientific literature.

While Walsh et al.'s ${ }^{2}$ findings were in line with those of this study (i.e., only $26.5 \%$ overlap with a condition-specific search for spinal cord injury), they came to a very different conclusion. Walsh et al. ${ }^{2}$ assert that the small amount of overlap between the two searches is not a problem because $98 \%$ of the articles that were not captured with the general search terms $(\mathrm{n}=$ 285) would not have met the criteria for inclusion in their systematic search. However, an alternative interpretation of this finding is that approximately 6 articles would have been missed related to this one specific condition if the systematic search strategy only includes their general 
disability search terms. Given that this current study suggests each condition-specific search results in only a small amount of overlap, it is likely these misses will compound. Therefore, using only Walsh et al.' ${ }^{2}$ general disability search terms is likely, in many cases, insufficient for obtaining the broad literature capture necessary for a systematic search and review.

While this study achieved the goal of providing insight into the search strategy necessary to capture sources related to opioids and disability, it is not without limitations. First, the results of this study are based on the utilization of three specific databases (i.e., Medline, CINHAL, PsycINFO). Therefore, the results of this study are directly applicable to these databases. However, these three data bases were utilized because they allow for a replication and extension of the work by Walsh et al. ${ }^{2}$ Additionally, these databases are three of the most commonly utilized databases in health-related systematic searches and reviews. Thus, our findings have implications for most other systematic searches in this domain. Another potential limitation is that translating the results of this study into practice will inherently increases the number of search results that have to be processed during a systematic search and review. Walsh et al. ${ }^{2}$ argue that the benefit of their search terms is that they provide a broad capture of the literature, while also limiting the number of search results that will need to be processed. While search strategies are most effective when optimized for efficiency, we found very little overlap between the results of the general disability search and the condition-searches conducted in this study. This included only $8.5 \%$ of the unique sources captured with the spinal cord injury search also being captured in the general disability search. Therefore, many relevant sources would have been missed in a search utilizing only the search terms proposed by Walsh et al. ${ }^{2}$

The findings of this study have several implications for other disability-related systematic searches. First, this study suggests that it may be necessary to utilize condition-specific search 
terms, even when the goal of a systematic search is to identify sources related broadly to people with disability (e.g., opioid use among people with disability). In practice, this means that researchers need to ensure they have created a detailed operational definition of the term "disability" in the context of their study before they conduct their formal systematic search. Otherwise, high-yield condition-specific terms may be left out of the search strategy protocol. Additionally, this means that researchers conducting systematic searches related to disability will need to be prepared to process a high number of search results. Software and other technology exists to help with this process, including software packages that track and eliminate duplicate sources, and provide a platform for efficiently making and reconciling include/exclude determinations. While this is still a time and resource intensive endeavor with these technological resources, this study demonstrates the importance of not attempting to reduce the burden of this work by utilizing only general disability search terms. Systematic searches, and the reviews and meta-analyses based on them, are most informative when they completely capture the scientific work related to a specific topic.

\section{Conclusion}

Our pilot systematic search study suggests that including condition-specific terms, at least in some cases, is necessary for ensuring the most complete capture of scientific literature. 


\section{References}

1. Schaefer N. Disability Search Tips and Resources. Med Ref Serv Q. 2015;34(1):60-74. doi:10.1080/02763869.2015.986792.

2. Walsh ES, Peterson JJ, Judkins DZ, Expert Panel on Health Care Disparities Among Individuals With Disabilities. Searching for disability in electronic databases of published literature. Disabil Health J. 2014;7(1):114-118. doi:10.1016/j.dhjo.2013.10.005. 
A.

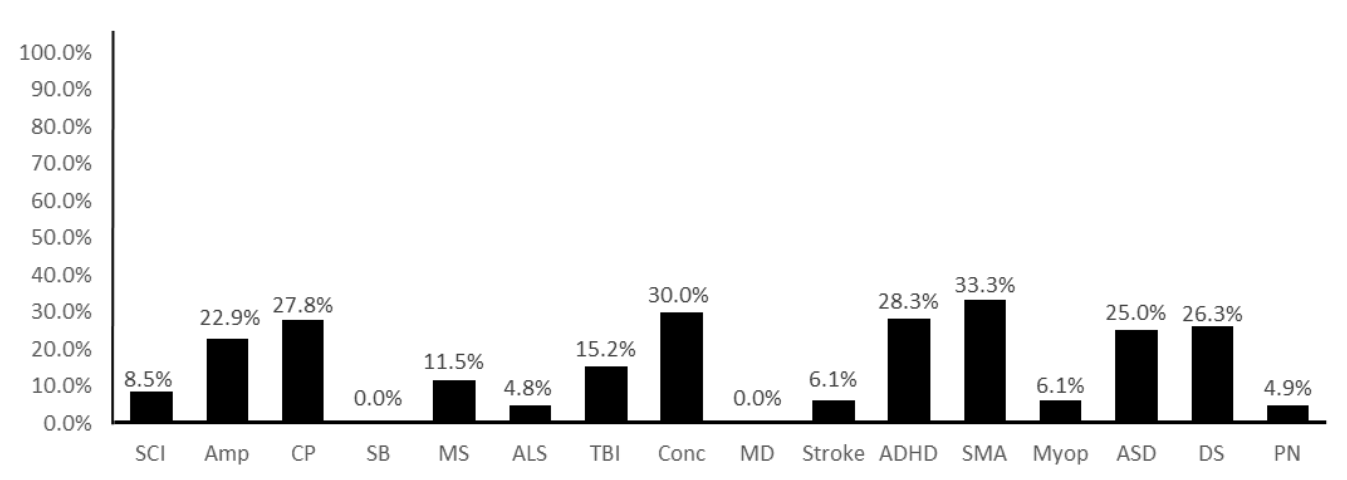

B.

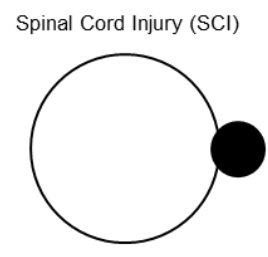

Spina Bifida (SB)

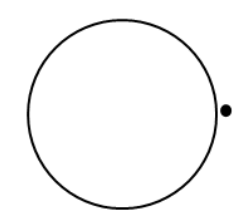

Traumatic Brain Injury (TBI)

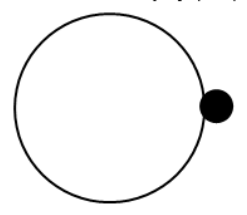

Myopathy (Myop)

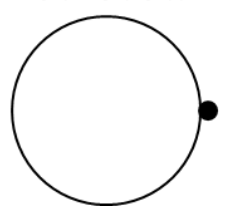

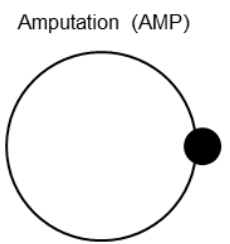

Multiple Sclerosis (MS)

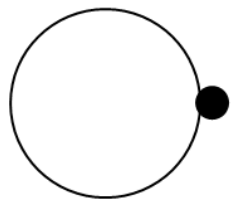

Concussion (Conc)

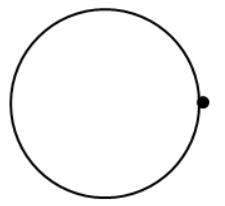

Autism Spectrum Disorder (ASD)

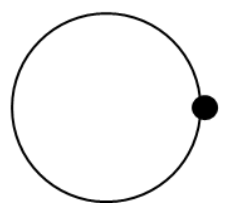

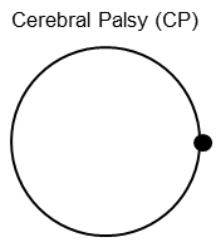

Amyotrophic Lateral Sclerosis (ALS)

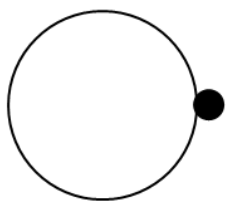

Muscular Dystrophy (MD)

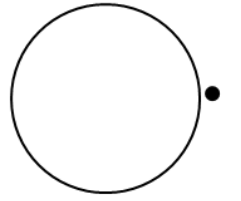

Down Syndrome (DS)

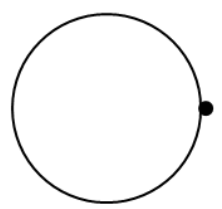

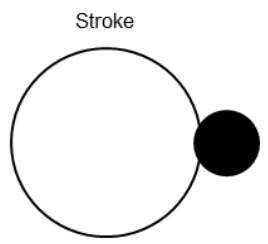

Peripheral Neuropathy (PN)

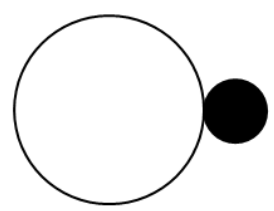

Spinal Muscular Atrophy (SMA)

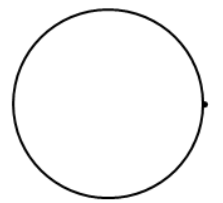

Attention-Deficit/ Hyperactivity Disorder (ADHD)

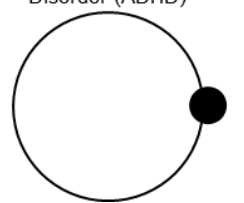

Figure 1. Overlap between each condition-specific search and the search utilizing the Walsh et al. disability search terms. A. A chart providing the percent of unique sources for each conditionspecific search that were also captured by the Walsh et al. disability search terms. B. A proportional visual representation of the Venn diagrams illustrating overlap between the unique sources found in each condition specific search (all black circles) and the search utilizing the Walsh et al. disability search terms (white circle with black outline). 
Table 1. Recommended General and Condition-Specific Search Terms

\begin{tabular}{|l|l|}
\hline General & Condition-Specific \\
\hline Disabled persons/ & SCI/ \\
Amputees/ & Amputation/ \\
Hearing impaired persons/ & Cerebral palsy/ \\
Mentally ill persons/ & Spina bifida/ \\
Visually impaired & Multiple sclerosis/ \\
persons/ & ALS/ \\
$\begin{array}{l}\text { Mentally Disabled } \\
\text { persons/ }\end{array}$ & Traumatic brain injury/ \\
“Activities of daily & Muscular dystrophy/ \\
living"/ & \\
$\begin{array}{l}\text { Developmental } \\
\text { disabilities/ }\end{array}$ & Stroke/ \\
Dependent ambulation/ & ADHD/ \\
Paraplegia/ & Doncussion/ \\
Quadriplegia/ & Spinal muscular \\
Hearing loss/ & atrophy/ \\
Blindness/ & Myopathy/ \\
Vision disorders/ & ASD/ \\
exp. Self-help devices/ & Peripheral neuropathy/ \\
Mental Disorders/ & \\
\hline \multicolumn{1}{|c|}{ Opioid Related Disorders } \\
\hline
\end{tabular}


Table 2. Number of Unique Sources for Each Search

\begin{tabular}{lrrrr}
\hline Specific Search & $\begin{array}{r}\text { Total Number of } \\
\text { Unique Sources }\end{array}$ & $\begin{array}{c}\text { Number of Unique } \\
\text { Sources }\end{array}$ & \multicolumn{2}{c}{$\begin{array}{c}\text { of Specific Search } \\
\text { Overlapping }\end{array}$} \\
\hline Walsh et al Terms & 3168 & - & - \\
SCI & 248 & 21 & $8.5 \%$ \\
Amp & 109 & 25 & $22.9 \%$ \\
CP & 18 & 5 & $27.8 \%$ \\
SB & 11 & 0 & $0.0 \%$ \\
MS & 96 & 11 & $11.5 \%$ \\
ALS & 83 & 4 & $4.8 \%$ \\
TBI & 92 & 14 & $15.2 \%$ \\
Conc & 10 & 3 & $30.0 \%$ \\
MD & 14 & 0 & $0.0 \%$ \\
Stroke & 375 & 23 & $6.1 \%$ \\
ADHD & 120 & 34 & $28.3 \%$ \\
SMA & 3 & 1 & $33.3 \%$ \\
Myop & 33 & 2 & $6.1 \%$ \\
ASD & 52 & 13 & $25.0 \%$ \\
DS & 19 & 5 & $26.3 \%$ \\
PN & 366 & 18 & $4.9 \%$ \\
\hline
\end{tabular}

